\title{
Factores de riesgo del parto prematuro en gestantes trabajadoras
}

\author{
Luz Marina Valencia B.*; Marta Lucía Vásquez T.**
}

El presente trabajo fue presentado en el II Congreso Colombiano de Perinatología celebrado en Cali del 31 de Mayo al 2 de Junio de 1991.

RESUMEN: Para determinar si el trabajo es un factor de riesgo en el parto prematuro (sin entidades asociadas), se estudiaron 53 partos prematuros y 160 partos normales, como controles, en la clínica del Instituto de Seguro Social en Cali Colombia. Los partos controles se debían producir en el mismo período del caso, dentro del rango de una semana. Estos partos se atendieron de noviembre 1989 a mayo 1990 . En el análisis multivariado de regresión logística, como factores de riesgo se encontraron tanto la exposición a vibraciones (coeficiente de regresión, 0.117302),

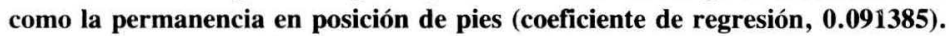

Estos hechos indican que es imperativo establecer protección de la salud y vigilancia en los lugares donde trabajan embarazadas. Así mismo es necesario fijar normas a los límites de exposición ocupacional y aplicar las medidas que permitan descubrir problemas de salud que se relacionen con el trabajo, para darles un tratamiento adecuado.

PALABRAS ClAVE: Parto prematuro, Mujer trabajadora, Derechos de la mujer, Exposición ocupacional, enfermedad ocupacional.

SUMMARY: To determine if working activities are risk factors in premature birth (with no other associated conditions), 53 premature deliveries and 160 normal labors were studied at the Social Security Institute clinic in Cali, Colombia. Control casos were recorded at the same time, within a range of one week. All of the deliveries occurred from November 1989 to May 1990 . At the logistic regression of multivariate analysis, as main risk factors, exposure to vibrations (regression coefficient, 0.117302), as well as the permanence of the standing up position (regression coefficient, $\mathbf{0 . 0 9 1 3 8 5}$ ) were found.

These data suggest the requirement of establishing health protection and vigilance at the working places of pregnant women. Also it is mandatory to constitute arrangements for the limits of occupational exposure and to give guidelines designed to detect health problems related both to the job itself and to the working place, in order to give them an adequate management.

KEY WORDS: Premature birth. Working women. Woman rights. Occupational diseases. Occupational exposure.

\section{Introducción}

Las mujeres constituyen aproximadamente un tercio de la fuerza laboral a nivel mundial. ${ }^{1}$ En Africa las encuestas hogareñas indican que las mujeres hacen las tres cuartas partes del trabajo agrícola, algunas mujeres trabajan en microempresas, actividades comerciales o productivas. En América Latina las mujeres constituyen más o menos el $40 \%$ de los trabajadores ${ }^{2}$, la fuerza de trabajo femenina en esta región se encuentra en ramas económicas como la agricultura $(73.79 \%)$, y la industria $(12.38 \%)$.

La inserción de la mujer en el proceso de trabajo adquiere particularidades muy propias en Latinoamérica, que no han sido estudiadas suficientemente desde el área de salud. En efecto, el perfil patológico de la trabajadora en la mayoría de los países de América Latina se desconoce.

Enfermera Magister en Enfermería materno-infantil. Secretaría Municipal de Salud de Cali.

** Enfermera Obstetriz. Magister en Epidemiología. Profesora Asociada Departamento de Enfermería Facultad de Salud Universidad del Valle.
Se puede suponer que la inmensa mayoría de mujeres están severamente afectadas a nivel orgánico y sicoemocional. Su articulación al proceso productivo, la doble jornada laboral, los riesgos laborales existentes, conforman un conjunto de factores que impactarían sus condiciones de vida en general y su estado de salud en particular.

La mujer embarazada tiene más riesgos laborales que la no embarazada ${ }^{3}$. El estado de gravidez induce modificaciones orgánicas que guardan cierta relación con las condiciones físicas maternas, el medio que las rodea, la edad y la estabilidad emocional; en muchas ocasiones no puede realizar sus funciones laborales, lo que afecta síquica, emocional y económicamente tanto a la futura madre como a su hijo y a la familia en general ${ }^{4}$.

La actividad laboral se caracteriza por riesgos físicos y químicos que tienen grandes repercusiones como aumento de proporción de abortos espontáneos, partos prematuros, y malformaciones fetales ${ }^{5,8}$.

En un gran número de partos prematuros se desconoce la etiología y además son difícilmente controlables los factores riesgo $0^{6,7}$. En algunos estudios se afirma que el tipo de 
trabajo realizado por las gestantes representa un factor de riesgo para desencadenar el parto prematuro ${ }^{7,8}$.

La morbilidad neonatal es mayor en los prematuros y se calcula que aproximadamente las $2 / 3$ partes mueren en la etapa neonatal temprana. Entre más corto sea el período gestacional más abundante será la patología del neonato ${ }^{9}$.

El parto prematuro representa entonces, un riesgo alto de mortalidad neonatal, y morbilidad materna, y afecta la estabilidad familiar y el estado emocional de la madre, lo cual muestra la magnitud del problema.

\section{Metodología}

Con el propósito de conocer si existe o no asociación entre el factor de riesgo, trabajo de la embarazada y el parto prematuro, se planteó un estudio de casos y controles en las mujeres en puerperio afiliadas y beneficiarias del Instituto de Seguros Sociales, hospitalizadas en la Clínica Rafael Uribe Uribe de Cali, desde Noviembre de 1989 hasta Mayo de 1990.

El tamaño calculado para la muestra fue de 54 casos y 162 controles. El cálculo del tamaño de la muestra, se basó en la fórmula de Schesselman $^{10}$, con un alfa $=0.05$, beta $=0.20$, poder 1 -beta $=0.80$, frecuencia de exposición en los controles de 0.5 y un riesgo relativo de 2.0 .

El caso debía ser gestante afiliada o beneficiaria del I.S.S. que hubiera tenido su parto en la Clínica Rafael Uribe de Cali y cuyo embarazo hubiera terminado sin causa orgánica demostrable entre las 21 y 36 semanas de gestación calculada por la amenorrea o por el método de cálculo de la edad del neonato, de Capurro.

Cada control debía cumplir con las siguientes condiciones:

- Ser gestante afiliada o beneficiaria del I.S.S. y haber tenido su parto en la Clínica Rafael Uribe Uribe de Cali.

- La culminación del embarazo debía haber ocurrido entre las 37 y las 42 semanas de gestación.

- La culminación del embarazo no debía ser ocasionada por causa orgánica demostrable.

- El parto debía haber ocurrido durante el mismo período del caso con un rango de una semana (la siguiente a la selección del caso).

La recolección de la información se llevó a cabo a través de una encuesta, que fue aplicada directamente a las gestantes seleccionadas para el estudio. Su aplicación y diligenciamiento tuvo una duración aproximada de 30 minutos y se aplicó durante el puerperio inmediato cuando la madre estaba aún hospitalizada en la Clínica Rafael Uribe Uribe de Cali, con el fin de evitar la pérdida de personas de la muestra y sesgos por olvido de la madre.

Las encuestas fueron realizadas por dos auxiliares de enfermería y una enfermera previamente capacitadas.

Para el procesamiento de datos, a cada respuesta se le asignó un código y se creó un archivo de datos DBase III plus. Se utilizó el paquete EPIINFO para el análisis estadístico básico y con el paquete SYSTAT se efectuó el análisis multivariado de regresión logística.

Un caso y dos controles fueron excluidos del análisis por encontrarse mal clasificados.

\section{Resultados}

El $77.4 \%$ de los casos y el $90 \%$ de los controles provenían de Cali, puesto que la Clínica Rafael Uribe Uribe representa el nivel I, II, II de atención y es el sitio donde por su infraestructura deben asistir toda las gestantes a tener su parto.

La edad de las madres fue homogénea tanto para el grupo de casos como el de controles, con una distribución ligeramente asimétrica con predominio de mujeres jóvenes, en el grupo de 20-34 años, (casos $83.0 \%$ y controles $80.0 \%$ ).

No se encontró asociación entre la edad y el parto prematuro $\left(\mathrm{X}^{2}=1.8 \quad \mathrm{P}=0.17\right)$.

Los porcentajes de alfabetismo fueron altos tanto para casos como para controles, con $98.1 \%$ y $100 \%$ respectivamente.

$\mathrm{Al}$ analizar la distribución según años de escolaridad aprobados se encontró que el $51.9 \%$ de los casos y el $57.9 \%$ de los controles estudiaron de 6-11 años.

El $25 \%$ de los casos y el $18.2 \%$ de los controles llevaron a cabo estudios superiores.

Tanto casos como controles mostraron porcentajes altos de unión estable ( $81.13 \%$ y $73.75 \%$ respectivamente).

El estrato social de las gestantes, se determinó mediante las categorías creadas por el I.S.S, basándose en los ingresos de las afiliadas y beneficiarias.

La categoría 20 es la catalogada como el sueldo mínimo $(\$ 38.040,00$ a $\$ 44.039,99)$ al momento del estudio y con base en esto se encontró que $43 \%$ de los casos y $45 \%$ de los controles entre afiliadas y beneficiarias ganaban menos del sueldo mínimo. $15 \%$ de los casos y $10 \%$ de los controles ganaban el sueldo mínimo. (Tabla 1)

\section{Tabla 1 \\ VARIABLES SOCIODEMOGRAFICAS DE GESTANTES INSTITUTO DE SEGUROS SOCIALES \\ CALI, 1990}

\begin{tabular}{|lllllr|}
\hline Variable & \multicolumn{2}{c}{ Resultados } & Casos & \multicolumn{2}{c|}{ Controles } \\
& & $\mathrm{n}$ & $\%$ & $\mathrm{n}$ & \multicolumn{1}{c|}{} \\
\hline Procedencia & Cali & 41 & 77.4 & 144 & 90.0 \\
Edad & $20-24$ & 44 & 83.0 & 128 & 80.0 \\
Escolaridad & Alfabetas & 52 & 98.1 & 160 & 100.0 \\
Convivencia & U.estable & 43 & 81.1 & 72 & 45.0 \\
\hline
\end{tabular}

Al análisis crudo se observó asociación entre parto pretérmino y gravidez de 5 embarazos anteriores $\mathrm{X}^{2}=5.8 \mathrm{P}=$ 0.02 ) con un ODDS RATIO de 6.45. (Tabla 2).

Hubo asociación estadística entre el antecedente de dos o más abortos y parto pretérmino $(\mathrm{Z}=4.1)(\mathrm{P}=0.00005)$. (Tabla 3).

$\mathrm{Al}$ analizar las variables del embarazo actual, se encontró que la mayoría de las gestantes tuvo al menos un control prenatal, se observó una distribución asimétrica con un riesgo relativo de 2.01 , indicando más probabilidad de parto prematuro con menos de 4 controles durante el embarazo $\left(\mathrm{X}^{2}=4.37 \quad \mathrm{P}=0.036\right)$. (Tabla 4)

En cuanto a la actividad laboral se analizaron antecedentes laborales y variables del trabajo actual.

En relación con empleos anteriores, se encontró que el 
Tabla 2

ANTECEDENTES DE GRAVIDEZ DE LAS GESTANTES INSTITUTO DE SEGUROS SOCIALES CALI, 1990

\begin{tabular}{|lccccc|}
\hline \multicolumn{2}{|c}{$\begin{array}{c}\text { Embarazos Casos } \\
\text { Anteriores n }\end{array}$} & $\%$ & $\mathrm{n}$ & $\%$ & \multirow{2}{*}{ Controles } \\
\hline 0 & 20 & 37.7 & 59 & 36.9 & 79 \\
1 & 13 & 24.5 & 55 & 34.4 & 68 \\
2 & 12 & 22.6 & 28 & 17.5 & 40 \\
3 & 3 & 5.7 & 12 & 7.5 & 15 \\
4 & 1 & 1.9 & 4 & 2.5 & 5 \\
$5^{*}$ & 4 & 7.5 & 2 & 1.3 & 6 \\
TOTAL & 53 & 100 & 160 & 100 & 213 \\
\hline
\end{tabular}

$\begin{array}{lll}\mathrm{X}^{2} & = & 5.8 \\ \mathrm{P} & = & 0.02 \\ \mathrm{D} . \mathrm{R} & = & 6.45 \\ & & \\ 1 . \mathrm{C}_{95 \%}=141-29.5 & \end{array}$

Tabla 3

ANTECEDENTES DE ABORTO INSTITUTO DE SEGUROS SOCIALES CALI, 1990

\begin{tabular}{|c|c|c|c|c|c|c|c|}
\hline Abortos & $\mathrm{n}^{\mathrm{C}}$ & s & $\begin{array}{c}\text { Controles } \\
n\end{array}$ & $\underset{\%}{\text { Total }}$ & $\mathbf{Z}$ & $\mathbf{P}$ & \\
\hline 0 & 23 & 69.7 & 73 & 72.3 & 96 & -0.3 & 0.77 \\
\hline 1 & 6 & 18.2 & 16 & 15.8 & 22 & 0.31 & 0.76 \\
\hline$>2$ & 4 & 12.1 & 12 & 11.9 & 16 & 4.1 & $0.00005^{*}$ \\
\hline TOTAL 3 & & 100 & 101 & 100 & 134 & & \\
\hline
\end{tabular}

Tabla 4

\section{NUMERO DE CONTROLES PRENATALES INSTITUTO DE SEGUROS SOCIALES CALI, 1990}

\begin{tabular}{|c|c|c|c|c|c|}
\hline $\begin{array}{l}\text { Control de } \\
\text { Embarazos }\end{array}$ & $\begin{array}{l}\text { Casos } \\
\mathrm{N}\end{array}$ & $\%$ & $\begin{array}{l}\text { Controles } \\
\mathrm{N}\end{array}$ & $\%$ & Total \\
\hline$<4$ & 26 & 49.1 & 51 & 31.9 & 77 \\
\hline$>4$ & 27 & 50.9 & 109 & 68.1 & 136 \\
\hline TOTAL & 53 & 100 & 160 & 100 & 213 \\
\hline
\end{tabular}

O.R. $=2.06$

I. $\mathrm{C}_{95 \%}=1.04-4.07$

$\mathrm{X}^{2}=4.37$

$\mathrm{P}=0.0364$

$35.2 \%$ de las gestantes de la muestra había trabajado antes del embarazo actual. Se observó asociación estadística entre haber trabajado antes del embarazo objeto de estudio y el parto prematuro $\left(\mathrm{X}^{2}=8.6, \mathrm{P}=0.0003\right)$.

La exposición a factores de riesgo ocupacional fue exposición a aire contaminado con polvo, temperaturas altas, temperaturas bajas, exposición a químicos, exposición a vibraciones, exposición a sobre - esfuerzo. posición de pies, exposición a radiaciones y exposición a ruidos. Se encontró asociación estadística entre la exposición a vibraciones y a la posición de pies y parto prematuro antes del embarazo y durante el mismo. (Tablas 5 y 6).

Mediante el análisis de regresión se encontró la exposición a vibraciones durante el embarazo, como factor de riesgo. Este fue de 1.02 lo que permite determinar que hay asociación entre la variable horas de vibración y ser caso con relación a efecto dosis, es decir, que entre más horas de exposición a la vibración tenga una mujer embarazada mayor será el riesgo de parto prematuro.

En relación con la exposición a la posición de pies durante el embarazo se observó un riesgo logarítmico de 0.009 de ser caso por cada hora de exposición a la posición de pies, encontrándose también una relación efecto dosis para la variable en mención. (Tabla 7)

Tabla 5

ANALISIS UNIVARIADO EXPOSICION A FACTORES DE RIESGO ANTES DEL EMBARAZO

\begin{tabular}{|c|c|c|c|c|c|c|c|}
\hline Factores de riesgo & $\begin{array}{l}\text { Casos } \\
\text { No. }\end{array}$ & $\%$ & $\begin{array}{l}\text { Contr } \\
\text { No. }\end{array}$ & $\begin{array}{r}\text { roles } \\
\%\end{array}$ & O.R. & $\mathbf{X}^{2}$ & $\mathbf{P}$ \\
\hline Polvo & 8 & 15.1 & 15 & 9.4 & 0.58 & 0.82 & 0.364 \\
\hline Temperaturas altas & 1 & 1.9 & 8 & 5.0 & 2.74 & 0.18 & 0.6676 \\
\hline Temperaturas bajas & 2 & 3.8 & 2 & 1.3 & 0.32 & 0.35 & 0.5556 \\
\hline Químicos & 3 & 5.7 & 4 & 2.5 & 0.43 & 0.45 & 0.500 \\
\hline Vibraciones & 3 & 5.7 & 0 & 0 & 0.00 & 2.56 & 0.014 a 2 colas* \\
\hline Sobreesfuerzo & 2 & 3.8 & 4 & 2.5 & 0.65 & 0.00 & 0.0046 \\
\hline Posición de pies & 19 & 35.8 & 32 & 20.0 & 0.45 & 4.66 & $0.0309 *$ \\
\hline Posición agachada & 3 & 5.7 & 7 & 4.4 & 0.76 & 0.00 & 0.992 \\
\hline Radiaciones & 1 & 1.9 & 0 & 0 & 0.00 & 0.34 & 0.2488 \\
\hline Ruido & 5 & 9.4 & 8 & 5.0 & 0.64 & 0.62 & 0.432 \\
\hline
\end{tabular}

Tabla 6

ANALISIS UNIVARIADO

EXPOSICION A FACTORES DE RIESGO DURANTE EL EMBARAZO

\begin{tabular}{|c|c|c|c|c|c|c|}
\hline \multirow[t]{2}{*}{ Factores de riesgo } & \multirow{2}{*}{$\begin{array}{l}\text { Casos } \\
\text { No. }\end{array}$} & \multirow[b]{2}{*}{$\%$} & \multicolumn{2}{|c|}{ Controles } & \multirow[t]{2}{*}{ O.R. } & \multirow[t]{2}{*}{$\mathbf{X}^{2}$} \\
\hline & & & No. & $\%$ & & \\
\hline Polvo & 6 & 11.3 & 13 & 8.1 & 0.69 & 0.180 .667 \\
\hline Temperaturas altas & 4 & 7.5 & 5 & 3.1 & 0.16 & 1.030 .3108 \\
\hline Temperaturas bajas & s 2 & 3.8 & 1 & 0.6 & 0.16 & 1.030 .3100 \\
\hline Químicos & 4 & 7.5 & 5 & 3.1 & 0.40 & 0.990 .3206 \\
\hline Vibraciones & 3 & 5.7 & 1 & 0.6 & 0.10 & 3.090 .048 a 2 colas* \\
\hline Sobreesfuerzo & 2 & 3.8 & 1 & 0.6 & 0.16 & 1.030 .310 \\
\hline Posición de pies & 17 & 32.1 & 26 & 16. & 0.41 & $5.250 .020 *$ \\
\hline Posición agachada & 4 & 7.5 & 6 & 3.8 & 0.60 & 0.570 .448 \\
\hline Radiaciones & 1 & 1.9 & 0 & 0 & 0.00 & 0.340 .56033 \\
\hline Ruido & 5 & 9.4 & 8 & 5.0 & 0.51 & 0.700 .40222 \\
\hline
\end{tabular}

Tabla 7

\begin{tabular}{|c|c|c|c|c|c|}
\hline Variable & Independiente & Coeficiente & $\begin{array}{c}\text { Error } \\
\text { estándar }\end{array}$ & $\begin{array}{c}95 \% \text { de } \\
\text { confianza }\end{array}$ & Límites \\
\hline Trabaja & 2 & -.1132276 & .8929474 & .1635929 & 4.8740201 \\
\hline Hora vibración & 1 & .2973505 & 1.3462871 & $5.994 \mathrm{e}-10$ & $3.024 \mathrm{e}+09$ \\
\hline Hora pié & 1 & -.0051066 & .9949064 & .9672972 & 1.0233036 \\
\hline Trabaja & 2 & 1.2012399 & 3.32422362 & .5629630 & 19.6292580 \\
\hline Hora vibración & 2 & .0117302 & 1.0117993 & .9547913 & 1.0722111 \\
\hline Hora pié & 2 & .0091385 & 1.0091804 & .9801613 & 1.0390586 \\
\hline
\end{tabular}




\section{Discusión}

Haciendo un análisis general de las variables sociodemográficas en gestantes afiliadas y beneficiarias, se puede destacar que los casos y controles son muy homogéneos en relación con la edad, alfabetismo, convivencia y estrato socioeconómico, lo que indica que la población en estudio posee similares factores sociodemográficos de riesgo para desencadenar partos prematuros.

A pesar de que en este estudio el parto prematuro no se encontró asociado a las variables sociodemográficas, algunas investigaciones muestran el alfabetismo, el nivel socioeconómico bajo y la unión inestable, como factores de riesgos potenciales para parto prematuro ${ }^{11,12}$.

Varios autores han encontrado que mujeres multigestantes que han tenido más de tres partos constituyen un factor de riesgo potencial para desencadenar parto prematuro ${ }^{13,7}$.

En cuanto a la asociación encontrada entre parto prematuro y el antecedente de dos o más abortos, se refuerza con resultados similares obtenidos por otro autor ${ }^{7}$, quien encuentra que el antecedente de aborto en embarazo anterior aumenta considerablemente el riesgo de hacer una amenaza de parto prematuro.

El factor de riesgo exposición a vibraciones durante el embarazo se encontró asociado al parto prematuro, observándose que las actividades laborales en las cuales estuvieron expuestas a vibraciones las gestantes del estudio fueron torneadora y jardinera (cortadora de pasto). Este resultado es similar al encontrado por Papierneck quien demostró que las embarazadas sometidas a vibraciones presentaron parto prematuro en un $26 \%$.

El otro factor de riesgo asociado al parto prematuro fue la exposición a la posición de pies durante el embarazo. Las ocupaciones más frecuentes, en las cuales las gestantes permanecieron en esta posición durante la gestación fueron vendedora ambulante y de mostrador, aseadora, tiqueteadora, profesora, operaria y mesera.

Algunos autores afirman que los diseñadores de máquinas no tienen en cuenta las necesidades de los seres humanos, sino el mayor rendimiento y, por lo tanto, la mayor productividad. Así por ejemplo: algunas trabajadoras deben permanecer en posición de pies continuamente, a lo cual se suma el estado de embarazo y a veces el calor, facilitando así, la aparición de varices, posiblemente afecciones inflamatorias y partos prematuros ${ }^{8.14}$.

Estos hallazgos sugieren como medida práctica y preventiva del parto prematuro, legislar sobre medidas que disminuyan la exposición a vibraciones y a la fatiga por posición de pies en las mujeres embarazadas. Por lo tanto, es importante fomentar la protección y vigilancia de la salud en los lugares de trabajo de las embarazadas mediante el establecimiento de límites de exposición profesional y la aplicación de medidas de detección y tratamiento apropiado de los problemas de salud relacionados con el trabajo.

\section{BIBLIOGRAFIA}

1. Population Crisis Commitee. Pobre, Desposeida y Embarazada. No. 20. Junio, 1988, p. 1-10.

2. Cuvi O., F. La mujer en las sociedades y continente Americano y su participación en el desarrollo. Publitécnica. Quito, 1983, p.302308.

3. Paredes, J. La mujer y el trabajo. Mimeografiado. 1986. p. 1-10.

4. Peña, M. La mujer, el trabajo y la maternidad. Material mimeografiado. p. 398.

5. OMS. Problemas de salud relacionados con el trabajo en condiciones de sobrecarga térmica. Informe de grupos científicos. Ginebra, 1969.

6. Schawrcz, Sala Duverages. Obstetricia. Tercera edición. Librería el Ateneo. Editorial Argentina. 1977.

7. Pasmiño, S. El parto prematuro en el ISS. Valle. En revista Colombiana Obstetricia y Ginecología. Vol. No. 2 p. 363-367, 1982. 367,1982

8. Desoiller, J.A; Martí Mecadal. Medicina del trabajo. Ministerio de la Cultura. Editorial Científico técnico. Ciudad de la Habana. Cuba, 1986. p.392-667.

9. Friedman, A., E. Obstetricia. Editorial Interamericana, Méjico, 1977

10. Schesselman, J. Cases and Controls studies. Oxford University Press 1982.

11. Cifuentes, B., Rodrigo. Obstetricia de alto riesgo. Talleres gracos de imprenta departamental. Tercera edición, 1990. p. 113-120.

12. Baird, D. Enviromental and obstetrical factors in premature with special reference to the experience in Aberdeen, Bulwha. 1981. p. 26-91

13. Grenhill, J.P. Emanuel, A. Obstetricia. Interamericana. Méjico, 1974. p. 685-688.

14. Epelman, M. La salud ocupacional de la mujer trabajadora. Salud y Trabajo, Ecuador. No. 5, 1983. p. 308-312. 